\title{
Seguridad y eficacia preliminar de las vacunas para la prevención de COVID-19
}

\author{
Preliminary safety and efficacy of vaccines for the prevention of COVID-19
}

\section{Comentado de:}

Perelli $L$, et al. Vacunas contra la COVID-19. Documentos de Evaluación de Tecnologías Sanitarias, Informe de Respuesta Rápida № 818, Buenos Aires, Argentina. 14 de enero de 2021. ISSN 1668-2793. Disponible en www.iecs.org.ar ${ }^{1}$

\section{Introducción}

La pandemia de COVID-19 ha generado un gran impacto en términos sanitarios, económicos, políticos y sociales en todo el mundo. La inmunidad de la población a través de la vacunación se plantea como la estrategia más racional para frenar la transmisión del coronavirus de tipo 2 causante del síndrome respiratorio agudo severo (SARS-CoV-2).

\section{Objetivo}

Describir las características que idealmente debería tener la vacunación contra la COVID-19, las diferentes tecnologías utilizadas en el desarrollo de estas vacunas que se encuentran en fases avanzadas de investigación clínica y datos preliminares de eficacia y seguridad.

\section{Métodos}

En el presente documento de Evaluación de Tecnologías Sanitarias, se recopila información proveniente de artículos científicos revisados o no revisados por pares, identificados en las principales bases de datos biomédicas, informes de plataformas de rastreo del desarrollo de vacunas y comunicaciones oficiales por parte de las empresas o institutos de investigación que lideran los ensayos de vacunas.

\section{Resultados}

Características que debería tener la vacunación para COVID-19

A fin de prevenir la enfermedad grave después de la infección, la vacunación debe dar lugar a: 1) la supresión o la reducción significativa de la transmisión dentro de la población mediante la inducción de la inmunidad del rebaño; y/o 2) la prevención de la enfermedad grave en los individuos vacunados. Una vacuna de dosis única que sea fácilmente distribuible y que no requiera equipos especializados y costos para mantener la cadena de frío contribuiría a que se pueda lograr una vacunación mundial a gran escala. Idealmente, la vacunación debería inducir una inmunidad de larga duración ${ }^{2}$.

Uno de los retos más importantes, que constituye una barrera para la inmunización primaria para diferentes enfermedades y una amenaza importante para la salud mundial, es la proliferación y el incremento en la visibilidad de grupos opositores a la vacunación, también llamados "antivacunas" ${ }^{3}$. Por este motivo se debe transparentar el proceso de evaluación de efectividad y seguridad de las vacunas.

El proceso de desarrollo de una vacuna suele durar una década, pero los plazos de COVID-19 se están reduciendo debido a la urgencia mundial por la pandemia, al tiempo que se intentan mantener las normas de seguridad y eficacia. Las fases involucradas en el desarrollo de nuevas vacunas, que pueden combinarse, se resumen en la Tabla 1.
Diferentes Instituciones y entidades académicas han desarrollado plataformas de seguimiento del desarrollo de vacunas contra la COVID- 19 (ver Tabla 2). Las vacunas que se encuentran en la actualidad en la fase 3 de investigación clínica se describen en la Tabla 3.

Tabla 1. Fases del desarrollo de las vacunas

\begin{tabular}{|l|l|}
\hline Fase & Descripción \\
\hline Preclínica & $\begin{array}{l}\text { Se realizan pruebas en animales para apoyar la } \\
\text { viabilidad y la seguridad. }\end{array}$ \\
\hline Clínica 1 & $\begin{array}{l}\text { Pequeño estudio que involucra entre 20 y 100 per- } \\
\text { sonas sanas, que evalúa la seguridad y la respues- } \\
\text { ta inmunológica en diferentes dosis. Típicamente } \\
\text { requiere un plazo de 1 a 2 años; en COVID 19 tomó } \\
\text { alrededor de 3 meses para algunas vacunas. }\end{array}$ \\
\hline Clínica 2 & $\begin{array}{l}\text { Estudia 100 a 300 personas. Evalúa más a fondo } \\
\text { la seguridad, evalúa la eficacia e informa sobre la } \\
\text { dosis óptima y el calendario de vacunación. Habi- } \\
\text { tualmente requiere plazos de 2 a 3 años, pero para } \\
\text { las pruebas de COVID-19, se espera que tome } \\
\text { alrededor de 8 meses. }\end{array}$ \\
\hline Clínica 3 & $\begin{array}{l}\text { Los científicos administran la vacuna a miles de } \\
\text { personas y esperan a ver cuántas se infectan, en } \\
\text { comparación con los voluntarios que recibieron un } \\
\text { placebo. Evalúa más a fondo la seguridad ya que } \\
\text { permite ver efectos adversos infrecuentes. Por lo } \\
\text { general se desarrolla en un plazo de de 2 a 4 años, } \\
\text { pero para los ensayos de COVID-19, puede combi- } \\
\text { narse con la fase ll. }\end{array}$ \\
\hline Revisión para & $\begin{array}{l}\text { Un organismo gubernamental examina los datos } \\
\text { de los estudios y la información presentada en la } \\
\text { solicitud de licencia antes de su aprobación. Esta } \\
\text { evaluación puede ocurrir mientras la fabricación ha } \\
\text { comenzado. De manera habitual se realiza en un } \\
\text { plazo de 1 a 2 años, pero para COVID-19, este pro- } \\
\text { ceso acelerado llevará unos pocos meses. }\end{array}$ \\
\hline $\begin{array}{l}\text { Estudios posteriores a la aprobación de la vacuna } \\
\text { que vigilan su eficacia y la seguridad en condicio- } \\
\text { nes reales. Las pruebas empiezan después de que } \\
\text { la vacuna se ha liberado al público. }\end{array}$ \\
\hline
\end{tabular}

Tabla 2. Plataformas de seguimiento del desarrollo de las vacunas para la COVID-19, disponibles en línea

\begin{tabular}{|l|l|}
\hline Entidad & Disponible en internet en el sitio \\
\hline $\begin{array}{l}\text { Organización Mun- } \\
\text { dial de la Salud }\end{array}$ & $\begin{array}{l}\text { https://www.who.int/publications/m/item/draft-la } \\
\text { ndscape-of-covid-19-candidate-vaccines }\end{array}$ \\
\hline $\begin{array}{l}\text { Escuela de Higiene } \\
\text { y Salud Tropical de } \\
\text { Londres }\end{array}$ & $\begin{array}{l}\text { https://vac-Ishtm.shinyapps.io/ncov_vaccine_la } \\
\text { ndscape/ }\end{array}$ \\
\hline Instituto Milken & https://www.covid-19vaccinetracker.org/ \\
\hline BioRender & https://biorender.com/covid-vaccine-tracker \\
\hline $\begin{array}{l}\text { The New York } \\
\text { Times }\end{array}$ & $\begin{array}{l}\text { https://www.nytimes.com/interactive/2020/scie } \\
\text { nce/coronavirus-vaccine-tracker.html }\end{array}$ \\
\hline
\end{tabular}




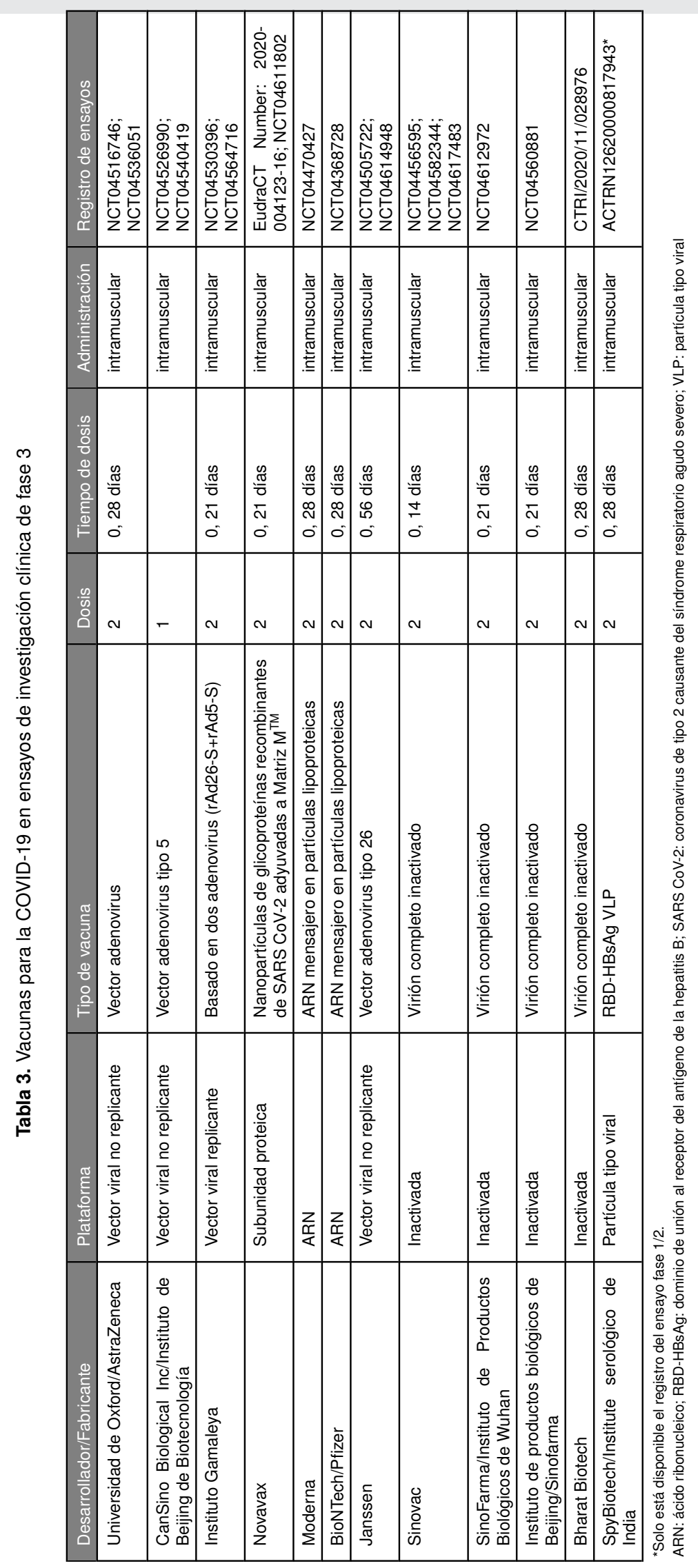


Vacunas con datos de eficacia clínica en estudios de fase 3

A continuación se resumen las características más relevantes de las vacunas contra el SARS-CoV-2 para las que se dispone evidencia proveniente de estudios clínicos de fase 3 .

\section{Gam-Covid-Vac o SPUTNIK-V}

El Instituto de Investigación Gamaleya forma parte del Ministerio de Salud de Rusia y ha producido la vacuna llamada GamCovid-Vac que utiliza como plataforma una combinación de dos adenovirus diferentes, Ad5 y Ad26, ambos diseñados con un gen de coronavirus. Para la conservación y distribución de esta vacuna se requiere refrigeración a $-18^{\circ} \mathrm{C}$. El 14 de diciembre de 2020 , el Instituto Gamaleya difundió datos del análisis final de los ensayos clínicos $(n=22.714 ; 17.032$ en el grupo vacuna y 5.682 en el grupo placebo). Se documentaron 78 casos (16 en el grupo vacuna y 62 en el grupo placebo), por lo que se estima una eficacia de la vacuna Sputnik V contra placebo de 91,4\% (intervalo de confianza [IC] del $95 \%$ de 85,1 a $95 \%$, calculado por los autores de este reporte) y un número necesario a tratar (NNT) de 100 (IC $95 \% 77$ a 143) en el día 21 o 28 después de la primera dosis (el segundo análisis interino con 18.794 casos fue reportado a 28 días, es decir 7 días después de la segunda dosis, pero en el análisis final, con 3.920 participantes adicionales, fue reportado a 21 días). La eficacia de la vacuna Sputnik V frente a casos graves de COVID-19 fue de $100 \%$ (20 casos graves en el grupo que recibió placebo, mientras que no se registraron casos graves en el grupo que recibió vacuna) ${ }^{4}$.

Con anterioridad se había comunicado una eficacia superior al $95 \% 42$ días después de la primera dosis (se corresponde con 21 días después de la segunda dosis) ${ }^{5}$. No se reportaron eventos adversos inesperados durante las pruebas. En su comunicado de prensa, el fondo ruso de inversión directa dijo que los resultados del ensayo se publicarían eventualmente en una revista científica revisada por pares.

El 24 de Diciembre de 2020 el Ministerio de Salud de la Nación Argentina autorizó con carácter de emergencia el uso de la vacuna Sputnik-V y notificó a la Administración Nacional de Medicamentos, Alimentos y Tecnología Médica (ANMAT), que previamente había recomendado mediante un informe técnico avanzar con esta autorización ${ }^{6,7}$. El informe técnico de ANMAT menciona que la información disponible muestra seguridad y una eficacia en un rango mayor al aceptable, e indica que no se han presentados eventos adversos graves, ni menor efectividad en los diferentes grupos etarios para los cuales está indicada actualmente (de 18 a 60 años de edad).

\section{Pfizer y BioNTech (BNT162b2)}

En mayo, Pfizer y BioNTech lanzaron un ensayo de fase 1/2 en dos versiones de una vacuna de ácido ribonucleico mensajero (ARNm) y encontraron que la llamada BNT162b2, producía significativamente menos efectos secundarios ${ }^{8,9}$. Esta vacuna requiere refrigeradores especiales que mantengan una temperatura de $-80^{\circ} \mathrm{C}$, lo que puede suponer un gran reto logístico para los países que deseen aplicarla.

El 18 de Diciembre su publicaron los resultados del ensayo clínico de fase 3 en el que se evaluó esta vacuna. Los investigadores enrolaron 43.548 participantes, de los cuales 43.448 recibieron inyecciones: 21.720 con BNT162b2 y 21.728 con placebo ${ }^{10}$. Hubo 8 casos de Covid-19 con fecha de inicio al menos 7 días después de la segunda dosis entre los participantes asignados a recibir BNT162b2 y 162 casos entre los asignados al placebo; por lo que se estableció que BNT162b2 fue 95,1\% efectiva en la prevención de COVID-19 (IC 95\% 90,3 a 97,6), con un NNT de 141 (IC $95 \% 125$ a 167). Se observó una eficacia similar de la vacuna (generalmente de 90 a $100 \%$ ) en todos los subgrupos definidos por edad, sexo, raza/etnia, índice de masa corporal de referencia y la presencia de condiciones coexistentes. Entre 10 casos de Covid-19 grave con inicio después de la primera dosis, nueve de ellos se produjeron en receptores de placebo y sólo uno, en un receptor de BNT162b2. En cuanto a la seguridad, se reportaron principalmente dolor a corto plazo (de leve a moderado, en el lugar de la inyección), fatiga y cefalea. No se observaron diferencias entre la vacuna y el placebo en cuanto a la incidencia de efectos adversos graves.

La Administración de Alimentos y Medicamentos de los Estados Unidos (FDA, por sus iniciales en inglés) y la ANMAT otorgaron la autorización de uso de emergencia a esta vacuna y su suministro para la inmunización activa con el fin de prevenir la enfermedad COVID-19 causada por el virus del SARS-CoV$2^{11,12}$

\section{Moderna (ARNm-1273)}

Moderna, junto al Instituto Nacional de Salud de los EE.UU. ( $\mathrm{NIH}$, por sus iniciales en inglés) desarrollaron una vacuna que también utiliza como plataforma el ARNm para producir proteínas de la espícula del SARS-CoV-2 frente a la cual se desencadena la respuesta inmune ${ }^{13}$. Esta vacuna es un ARNm que codifica una versión modificada de glicoproteínas de la espícula SARS-CoV-2. El ARNm se encapsula en nanopartículas lipídicas a una concentración de $0,5 \mathrm{mg}$ por mililitro y se diluye con solución salina normal para alcanzar las concentraciones de la vacuna final. Este ARNm contiene instrucciones genéticas para construir la proteína de la espícula del coronavirus. Esta vacuna requiere de refrigeración a $-20^{\circ} \mathrm{C}$ para su distribución.

El 30 de Diciembre de 2020 se publicaron los resultados del estudio de fase 3 de esta vacuna ${ }^{14}$. En este estudio se reclutaron 30.420 voluntarios que fueron asignados al azar en una proporción de 1:1 para recibir la vacuna o el placebo (15.210 participantes en cada grupo). Más de $96 \%$ de los participantes recibieron ambas inyecciones, mientras que 2,2\% presentó evidencia (serológica, virológica o ambas) de infección por SARS-CoV-2 al inicio del estudio. Se confirmó la COVID-19 sintomática en 185 participantes del grupo de placebo (56,5 por 1000 personas-año; IC $95 \%, 48,7$ a 65,3 ) y en 11 participantes del grupo de ARNm-1273 (3,3 por 1000 personas-año; IC $95 \%, 1,7$ a 6,0), por lo que la eficacia de la vacuna se estimó en 94,1\% (IC 95\%, 89,3 a 96,8\%), con un NNT de 87 (IC $95 \% 77$ a 100). La eficacia fue similar en los principales subgrupos, incluida la evaluación 14 días después de la primera dosis, los análisis que incluyeron a los participantes que tenían pruebas de infección por SARS-CoV-2 al inicio, y los análisis en participantes de 65 años de edad o más. Se documentó COVID-19 grave en 30 participantes y un fallecido en el grupo placebo. En cuanto a la seguridad, se produjeron reacciones de hipersensibilidad moderada y transitoria después de la vacunación, con mayor frecuencia en el grupo de ARNm-1273. Los eventos adversos graves fueron raros, y su incidencia, similar en los dos grupos. Esta vacuna se encuentra autorizada para su uso de emergencia por la FDA desde el 17 de diciembre de $2020^{15}$.

\section{Astra Zeneca-Oxford (AZD1222 o ChAdOx1)}

La compañía británico-sueca AstraZeneca y la Universidad de Oxford (mediante su empresa derivada, Vaccitech) desarrollaron una vacuna (ChAdOx1) de vector viral no replicante que utiliza un adenovirus de chimpancé con deficiencia en su replicación y que contiene el material genético de la proteína de punta del virus de SARS-CoV-2 ${ }^{16-18}$. Esta vacuna puede almacenarse, transportarse y manipularse a $2-8{ }^{\circ} \mathrm{C}$ durante al menos seis meses.

El 19 de noviembre de 2020, los investigadores publicaron los primeros hallazgos de los ensayos de fase $2 / 3$ en el Reino Unido. Estudiaron a 160 personas de 18 a 55 años de edad, 160 entre 
56 y 69 años, y 240 de 70 años o más. No observaron ningún efecto secundario grave a ninguna edad.

El 23 de noviembre, anunciaron que un análisis preliminar de su ensayo de fase 3 , basado en el estudio de los primeros 131 casos de Covid-19 en las pruebas en el Reino Unido y Brasil, reveló que la vacuna era hasta $90 \%$ efectiva, dependiendo de la dosis ${ }^{19,20}$. Este análisis interino conjunto incluyó datos del ensayo de fase $2 / 3$ de COV002 en el Reino Unido y del ensayo de fase 3 de COV003 en Brasil, y fue publicado el 8 de diciembre de $2020^{21}$. Evaluaron a 23.848 participantes siguiendo dos dosis de un régimen de media dosis/dosis completa o un régimen de dos dosis completas de AZD1222 o un comparador, la vacuna meningocócica conjugada llamada MenACWY o una solución salina, en adultos de 18 años de edad o más, de diversas etnias y regiones geográficas, sanos o con comorbilidades estables. El régimen de dosificación estándar $(n=8.895)$ mostró una eficacia de $62 \%$ cuando se administró en dos dosis completas aplicadas con un mes de diferencia. Un hallazgo llamativo y reportado en el estudio fue que el régimen de dosificación de mitad de la dosis inicial de la vacuna, seguida de una dosis completa con un mes de diferencia $(n=2.741)$ mostró una eficacia de la vacuna de $90 \%$. Realizaron, además, un análisis combinado de ambos regímenes de dosificación ( $n=11.636$ ) que dio como resultado una eficacia media de $70 \%$. Todos los resultados fueron estadísticamente significativos ( $p$-valor menor a 0,01$)$. El análisis preliminar también mostró que la vacuna no sólo redujo los casos de COVID-19 sintomáticos sino que también redujo el número de casos asintomáticos, lo que puede suponer eficacia en la reducción de la transmisión del virus de persona a persona. No se informaron hospitalizaciones ni casos graves de la enfermedad en los participantes que recibieron la vacuna.

El 30 de diciembre de 2020 la ANMAT, mediante la resolución 9271/20 autorizó el registro de emergencia de esta vacuna ${ }^{22}$.

\section{Vacunación en Argentina}

El 29 de diciembre de 2020 se inició la campaña de vacunación en Argentina con la vacuna Gam-Covid-Vac o SPUTNIK V, y el 31 de diciembre del mismo año el Ministerio de Salud de la Nación presentó el documento "Lineamientos técnicos para la Campaña Nacional de Vacunación contra la COVID-19 en Argentina" 23,24.

Para esta campaña se establecieron como prioritarias para la vacunación aquellas personas en grupos de riesgo por exposición y función estratégica (en primer lugar, personal de salud, posteriormente, de las Fuerzas Armadas, de seguridad y personal de servicios penitenciarios; luego, personal docente y no docente) y según el riesgo de enfermedad grave (adultos de 70 o más años de edad y personas mayores residentes en hogares de larga estancia, luego adultos de 60 a 69 años, y posteriormente, adultos 18 a 59 años de edad que pertenezcan a grupos en riesgo). En cuanto a la vigilancia de seguridad en vacunas contra la COVID-19, se definió como "evento supuestamente atribuible a la vacunación e inmunización" (ESAVI) a cualquier situación de salud inesperada (signo no favorable o no intencionado, hallazgo anormal de laboratorio, síntoma o enfermedad) que ocurra posterior a la vacunación, aunque no necesariamente tiene una relación causal con la vacunación o con el producto biológico. Se estableció la notificación obligatoria todos los errores de vacunación (como dosis incorrecta o sitio no apropiado de administración) y de todos aquellos eventos adversos que impliquen hospitalización, riesgo de vida de la persona, discapacidad o desenlaces fatales.

El ministerio de salud estableció, además, que se realizarán informes periódicos de vigilancia de seguridad en vacunas y que se publicarán en su sitio web https://www.argentina.gob.ar /coronavirus/vacuna/equipos-salud/informes-seguridad. Hasta el 8 de enero de 2021, se notificaron 3.453 ESAVI posteriores a la vacunación tras la aplicación de 89.576 dosis ${ }^{25}$. De estos ESAVI, 99,3\% de los eventos reportados fueron leves y moderados, mientras que 25 casos fueron hospitalizados para tratamiento sintomático con recuperación.

\section{Esquemas alternativos de vacunación en discusión por la escasez} de vacunas

En medio de las crecientes olas de contagios por coronavirus, algunos países están intentando extender los limitados suministros de vacunas COVID-19 reduciendo las dosis o cambiando los esquemas de vacunación evaluados en los ensayos clínicos. Sin embargo, los datos sobre el impacto de tales medidas son escasos y las opiniones de los científicos están divididas sobre si se justifican los riesgos ${ }^{26}$.

El 30 de diciembre, el Reino Unido anunció que permitiría administrar dosis de dos diferentes vacunas contra el coronavirus con un intervalo de hasta 12 semanas, aunque, en ensayos clínicos, el intervalo fue de tres semanas. Al retrasar la segunda dosis, el gobierno espera disponer de más dosis para que más personas reciban la primer inoculación. Por otro lado, existe la posibilidad que se genere memoria inmunológica y una mejor respuesta al refuerzo.

La política actual de los EE.UU. es garantizar a los destinatarios de la vacuna una segunda inyección. El jefe del esfuerzo de vacunación contra el coronavirus Operation Warp Speed (que en español significa 'Operación a la Velocidad de la Luz'), ha sugerido que una vacuna, desarrollada por Moderna, podría administrarse a la mitad de la dosis utilizada en su mayor ensayo clínico. Sin embargo el jefe de la FDA, en un comunicado publicado el 4 de enero de este año, afirmó que sugerir cambios a los esquemas aprobados por esta entidad es prematuro y no está basado en la evidencia disponible. Por su parte la Organización Mundial de la Salud (OMS) recomendó esperar no más de seis semanas entre la primera y la segunda dosis de la vacuna Pfizer. Algunos investigadores también están preocupados por el impacto del intervalo de dosificación más largo en el propio coronavirus. Las personas que reciben una sola dosis de una vacuna de ARN producen niveles relativamente bajos de anticuerpos, y podrían aparecer variantes virales resistentes a las vacunas, aunque si las dosis únicas de vacuna redujeran la cantidad de infecciones naturales, también podrían reducir el riesgo de desarrollar resistencia. De todos modos, aunque algunas variantes fueran parcialmente resistentes a las vacunas, es poco probable que este fenómeno genere que las vacunas se tornen completamente ineficaces.

En última instancia, cada país deberá tomar una decisión basada en sus propias necesidades y contexto.

\section{Comentario}

En el presente documento se recopila información proveniente de artículos científicos revisados o no por pares, informes de diferentes plataformas de rastreo del desarrollo de vacunas y comunicaciones oficiales por parte de las empresas o institutos de investigación que lideran los ensayos de las potenciales vacunas en estudio contra el SARS-CoV-2. Se describen los datos preliminares de eficacia y seguridad de los ensayos en fase 3 de las vacunas desarrolladas por el Instituto de Investigación 
Gamaleya, Pfizer-BioNTech, Moderna y Astra Zeneza-Oxford, a partir de la recopilación de comunicaciones oficiales. Se presenta el estado de la Campaña Nacional de Vacunación contra la COVID-19 en Argentina y la discusión planteada a nivel global sobre esquemas alternativos de vacunación para hacer frente a la escasez de vacunas.

Los datos preliminares provenientes de ensayos clínicos en fase 3 muestran resultados alentadores, con eficacias que rondan o superan el $90 \%$ y con un perfil de seguridad aceptable durante el tiempo de seguimiento disponible. Aunque cabe destacar que ningún estudio ha completado estos ensayos -que tomarán meses- y algunos no han reportado aun los reportes preliminares en revistas con revisión de pares.

Si bien la comunidad científica espera con ansias todas las publicaciones, no deberíamos sobrestimar el valor de la revisión de pares que repetidamente ha demostrado ser falible 27,28 , sino darle la jerarquía científica que se merece. Los organismos regulatorios de diversos países con esta información, han emitido aprobaciones de emergencia.

\section{Conclusiones del comentador}

No hay evidencia a la fecha que indique la superioridad de una vacuna para el SARS-CoV-2 por sobre la otra y hasta se están considerando, en algunos países, la posibilidad de completar esquemas con vacunas diferentes. Debido a que la pandemia sigue golpeando crudamente la salud y la economía mundial, a pesar de las posibles limitaciones de la evidencia resumida, la comunidad toda debiera abrazar la vacunación masiva para minimizar los cuantiosos daños ocasionados por este virus. Existen fuerte imperativos éticos y morales para cuidar no sólo nuestra salud individual, sino también la de nuestros pares, en particular la de los grupos más vulnerables.

Agustín Ciapponi [ Servicio de Medicina Familiar y Comunitaria, Hospital Italiano de Buenos Aires. agustin.ciapponi@hospitalitaliano.org.ar ]

Ciapponi A Seguridad y eficacia preliminar de las vacunas para la prevención de COVID-19. Evid Actual Pract Ambul. 2021;24(1):e002116. Comentado de: Perelli L, et al. Vacunas contra la COVID-19. Documentos de Evaluación de Tecnologías Sanitarias, Informe de Respuesta Rápida № 818, Buenos Aires, Argentina. 14 de enero de 2021. ISSN 1668-2793. Disponible en www.iecs.org.ar

\section{Referencias}

1. Perelli L, Martí SG, Alfie V, et al.. Documentos de Evaluación de Tecnologías Sanitarias, Informe de Respuesta Rápida № 818. Buenos Aires Argentina: Instituto de Efectividad Clínica y Sanitaria; 2021. Available from: Disponibleenwww.iecs.org.ar.

2. Hodgson SH, Mansatta K, Mallett G, Harris V, Emary KRW, Pollard AJ. What defines an efficacious COVID-19 vaccine? A review of the challenges assessing the clinical efficacy of vaccines against SARS-CoV-2. The Lancet Infectious Diseases. 2020;Available from: 10.1016/s1473-3099(20) 30773-8;https://dx.doi.org/10.1016/s1473-3099(20)30773-8.

3. World Health Organization. Ten threats to global health in 2019; 2019. Available from: https://www.who.int/news-room/spotlight/ten-threats-to-globalhealth-in-2019 [Last access: 2021-01-26]

4. $4 \%$ efficacy for the Sputnik $V$ vaccine on day 28 after the first dose; vaccine efficacy is over $95 \% 42$ days after the first dose; 2020 . Available from: https://sputnikvaccine.com/newsroom/pressreleases/second-interim-analysis-of-clinical-trial-data-showed-a-91-4-efficacy-for-the-sputnik-vvaccine-on-d/ [Last access: 2021-01-26]

5. The Sputnik V vaccine's efficacy is confirmed at $91.4 \%$ based on data analysis of the final control point of clinical trials ; 2020 . Available from: https://sputnikvaccine.com/newsroom/pressreleases/the-sputnik-v-vaccine-s-efficacy-is-confirmed-at-91-4-based-on-data-analysis-ofthe-final-control-po/ [Last access: 2021-01-26].

6. A M. Argentina. Ministerio de Salud, Secretaría de Calidad en Salud. Informe técnico de perfil regulatorio sobre autorización de uso de vacuna Sputnik V en el marco de la Ley 27.573; 2020. Available from: https://www.argentina.gob.ar/sites/default/files/if-2020-89983542-apn-anmatms.pdf.

7. Argentina. Ministerio de Salud. Resolución 2784/2020. ; 2021. Available from: https://www.boletinoficial.gob.ar/detalleAviso/primera/239160/ 20201224.

8. Walsh EE, Frenck RW, Falsey AR, et al. Safety and Immunogenicity of Two RNA-Based Covid-19 Vaccine Candidates. N Eng J Med. 2020;383(25):2439-2450. Available from: 10.1056/nejmoa2027906.

9. Mulligan MJ, Lyke KE, Kitchin N, et al. Phase I/II study of COVID-19 RNA vaccine BNT162b1 in adults. Nature. 2020;586(7830):589-593. Available from: 10.1038/s41586-020-2639-4.

10. Polack FP, Thomas SJ, Kitchin N, et al. Safety and efficacy of the BNT162b2 mRNA Covid-19 vaccine. N Engl J Med. 2020;383(27):2603-2615. Available from: 10.1056/NEJMoa2034577.

11. Food and Drug Administration. Emergency Use Authorization (EUA) for an Unapproved Product: Pfizer-BioNTech COVID-19 Vaccine/ BNT162b2 2020. Available from: https://www.fda.gov/media/144416/download.

12. Argentina. ANMAT. Resolución 9210-2020. 2020;Available from: https://www.argentina.gob.ar/sites/default/files/dispo_9210-2020.pdf.

13. Jackson LA, Anderson EJ, Rouphael NG, et al. An mRNA vaccine against SARS-CoV-2-preliminary report. N Engl J Med. 2020;383(20):1920-1931. Available from: 10.1056/NEJMoa2022483.

14. Baden LR, Sahly E, Essink HM, et al. Efficacy and safety of the mRNA-1273 SARS-CoV-2 vaccine. N Engl J Med. 2020;Available from: 10.1056/ NEJMoa2035389.

15. Vaccines and Related Biological Products Advisory Committee December 17, 2020 Meeting Presentation-Emergency Use Authorization (EUA) Application for MRNA-1273 Emergency Use Authorization (EUA) Application for MRNA-1273; 2020. Available from: https://www.fda.gov/media/ 144434/download.

16. Folegatti PM, Bittaye M, Flaxman A, et al. Safety and immunogenicity of a candidate Middle East respiratory syndrome coronavirus viral-vectored vaccine: a dose-escalation, open-label, non-randomised, uncontrolled, phase 1 trial. Lancet Infect Dis. 2020;20(7):816-826. Available from: 10.1016/ S1473-3099(20)30160-2.

17. Phase III Double-blind, Placebo-controlled Study of AZD1222 for the Prevention of COVID-19 in Adults; 2020 . Available from: https://clinicaltrials. gov/ct2/show/NCT04516746.

18. Doremalen NV, Lambe T, Spencer A, et al. ChAdOx1 nCoV-19 vaccine prevents SARS-CoV-2 pneumonia in rhesus macaques. Nature. 2020;586(7830):578-582. Available from: 10.1038/s41586-020-2608-y.

19. AZD1222 vaccine met primary efficacy endpoint in preventing COVID-19; 2020. Available from: https://www.astrazeneca.com/media-centre/pressreleases/2020/azd1222hlr.html [Last access: 2021-01-26] 
20. Oxford University breakthrough on global COVID-19 vaccine; 2020. Available from: https://www.research.ox.ac.uk/Article/2020-11-23-oxforduniversity-breakthrough-on-global-covid-19-vaccine [Last access: 2021-01-26].

21. Voysey M, Clemens SAC, Madhi SA, et al. Safety and efficacy of the ChAdOx1 nCoV-19 vaccine (AZD1222) against SARS-CoV-2: an interim analysis of four randomised controlled trials in Brazil, South Africa, and the UK. Lancet. 2021;397(10269):99-111. Available from: 10.1016/S0140-6736(20) $32661-1$.

22. Argentina. ANMAT. Resolución 9271-2020; 2020. Available from: https://www.argentina.gob.ar/sites/default/files/anmat disposicion 9271-2020.pdf.

23. Argentina. Ministerio De Salud. Dirección de enfermedades inmunoprevenibles. Lineamientos técnicos para la Campaña Nacional de Vacunación contra la COVID-19; 2020. Available from: https://bancos.salud.gob.ar/sites/default/files/2020-12/covid19-lineamientos-tecnicos-para-campanianacional-de-vacunacion-contra-covid19.pdf.

24. Comenzó la campaña de vacunación contra COVID-19 en Argentina; 2021. Available from: https://www.argentina.gob.ar/noticias/comenzo-lacampana-de-vacunacion-contra-covid-19-en-argentina [Last access: 2021-01-26].

25. Argentina. Ministerio de Salud. Campaña Nacional de Vacunación contra la COVID-19. $3^{\circ}$ Informe de vigilancia de seguridad en vacunas; 2021. Available from: https://www.argentina.gob.ar/sites/default/files/3er_informe_seguridad-14-1-2021.pdf.

26. Ledford H. How can countries stretch COVID vaccine supplies? Scientists are divided over dosing strategies. Nature. 2021;589(7841):182. Available from: 10.1038/d41586-021-00001-6.

27. Ciapponi A. Del escándalo del Tamiflu a una revolución de la evidencia científica en salud. Evid Act Práct Ambul. 2014;17(2):42-45. Available from: http://evidencia.org/index.php/Evidencia/article/view/6295.

28. Ciapponi A. La cara oculta del estudio 329 y la manipulación de la evidencia científica. Evid Act Pract Ambul. 2016;19(3):71-75. Available from: http://www.evidencia.org/index.php/Evidencia/article/view/6444. 